\section{An apparatus for the production of circularly polarised vuv radiation}

\author{
U Heinzmann \\ Physikalisches Institut der Universität Münster, \\ Schlossplatz 7, 4400 Münster, West Germany
}

Received 11 February 1977, in final form 4 May 1977

Abstract An apparatus for the production of circularly polarised vacuum ultraviolet (VUV) radiation up to $9 \mathrm{eV}$ is described. A photon beam of high intensity and polarisation is used for the photoionisation of atomic and molecular vapour beams in order to obtain spin-polarised photoelectrons. The radiation source and the polariser are discussed in detail. Results of intensity and polarisation measurements are presented.

\section{Introduction}

Recent theoretical and experimental investigations have shown that spin-polarised electrons can be obtained by the photoionisation of alkali atoms with circularly polarised light (Fano effect: Fano 1969; for a review of this field see Kessler 1976). This conspicuous effect of spin-orbit coupling prompted. strong interest in the question of whether other elements also yield polarised photoelectrons. With the exception of the alkalis, atoms and almost all molecules have photoionisation thresholds which lie in the vacuum ultraviolet range (VUV). The intensity of vUV radiation which can be obtained in practice is always much smaller than the intensity of UV light produced, for example, by a xenon high-pressure lamp (Heinzmann et al 1970). It was therefore necessary to build a new apparatus with a high overall efficiency for the study of the Fano effect with vUV radiation. Spin polarisation measurements of electrons produced by the photoionisation of thallium (Heinzmann et al 1975, 1976) and lead atoms (Heinzmann 1977) have already been obtained with this apparatus.

It is the purpose of the present paper to describe the design and properties of a source of circularly polarised vuv radiation which yields a high-intensity, polarised photon beam up to an energy of $9 \mathrm{eV}$, and a schematic diagram of the apparatus is shown in figure 1 . The circularly polarised vuv radiation beam intersects the atomic or molecular yapour beam, after which the polarisation of the light is analysed. The photoelectrons produced are extracted by an electric field and are focused by electron optical components before being accelerated to $120 \mathrm{keV}$. Their spin polarisation is then determined with a Mott detector.

2 Design and calibration of the radiation source and detector In order to obtain a light source with very high intensity in the vUV range, the Hinteregger lamp was selected (for a review of

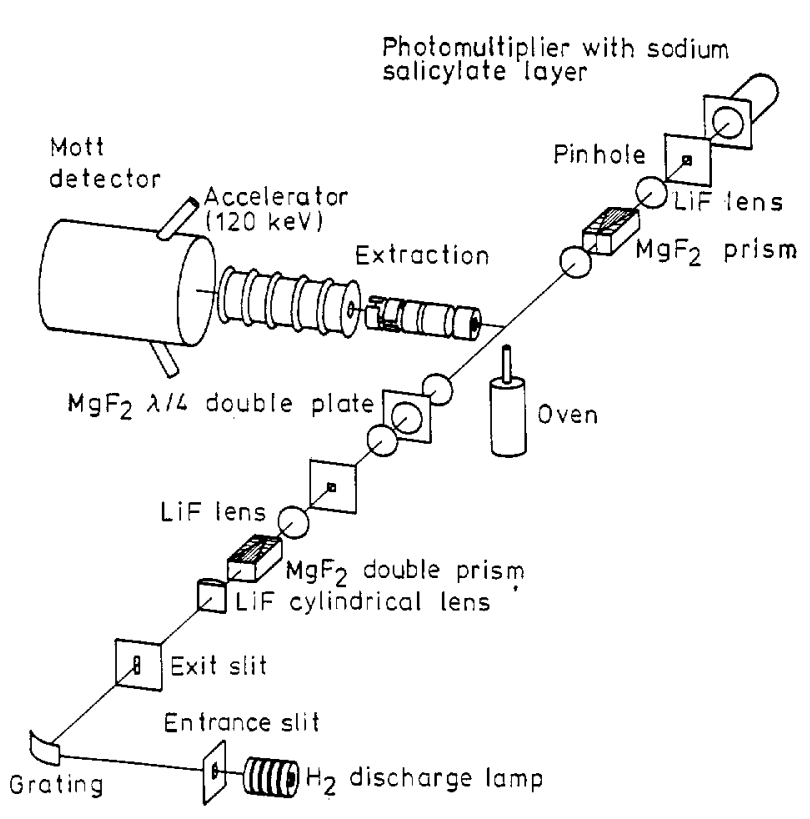

Figure 1 Diagram of the apparatus.

radiation sources, see Comes 1960). This lamp can run with different gases excited by a discharge as they stream through a silica tube (Huffman et al 1961, 1965); it can be used either with or without a window. In the apparatus described, an $\mathrm{MgF}_{2}$ window was used so that the monochromator entrance slit could be opened as wide as possible to obtain the highest photon flux. The monochromator was a Seya Namioka vUv type (McPherson model 235), which has an $\mathrm{MgF}_{2}$-coated concave grating ( $50 \mathrm{~mm} \times 30 \mathrm{~mm}, 0.5 \mathrm{~m}$ radius) with 600 lines $\mathrm{mm}^{-1}$ blazed at $150 \mathrm{~nm} . \mathrm{MgF}_{2}$ was preferred because uncoated or LiF-coated gratings slowly lose their high efficiency (Adriaens and Feuerbacher 1971).

For the calibration of the monochromator and for the spectral intensity measurements, the radiation detector, which is normally placed behind the light polarisation analyser (see figure 1), was mounted directly at the exit slit of the monochromator. For detection of the VUV radiation, the technique involving fluorescence of sodium salicylate was selected (Becker 1963, Branch et al 1971, Samson and Hadded 1974). This method was preferred to the use of a channeltron (Johnson 1969, Paresce 1975), a thermopile (Johnston and Madden 1965 ) or a photomultiplier with an LiF window and a caesium telluride cathode (Davis and Braun 1968) because of its ease of use and the constant quantum yield for varying wavelength and intensity. Methanol, in which the sodium salicylate has been dissolved, is slowly sprayed by a medicinal at omiser on to a glass plate at a distance of $50 \mathrm{~cm}$. A heat gun volatilises the methanol before it reaches the plate. A homogeneous sodium salicylate layer with a thickness of about $1 \mathrm{mg} \mathrm{cm}-2$ is formed in about $2 \mathrm{~h}$. The fluorescence light produced passes through an interference filter ( $424 \mathrm{~nm}$, halfwidth $19 \mathrm{~nm}$ ) and several neutral filters and is detected by the photocathode (S11, EMI) of a photomultiplier.

The wavelength scale of the vUV monochromator has been calibrated by the 253.7 and $455.6 \mathrm{~nm}$ resonance lines of $\mathrm{Hg}$ and $\mathrm{Cs}$, respectively, in the first and zero orders by replacing the Hinteregger lamp with an $\mathrm{Hg}$ or $\mathrm{Cs}$ low-pressure resonance lamp. The accuracy of the calibration, performed with $10 \mu \mathrm{m}$ slits, was $\pm 0.03 \mathrm{~nm}$. Furthermore, by using the same resonance lines the dispersion of the monochromator was found to be $1 \mathrm{~mm} / 3 \cdot 1 \mathrm{~nm}$. This means that the full width of the tri- 


\section{U Heinzmann}

angular intensity distribution at half-height measured behind the exit slit on rotating the grating is $3.1 \mathrm{~nm}$ if both slits are opened to a width of $1 \mathrm{~mm}$ (Christensen and Potter 1963). This halfwidth is the same as the bandwidth of continuous light leaving the monochromator, i.e. $3 \cdot 1 \mathrm{~nm}$, if the entrance and the exit slit are also opened $1 \mathrm{~mm}$.

\section{Power and spectrum of the radiation source}

The spectral intensities of the radiation coming from the Hinteregger lamp have been measured with different filling gases at different pressures and have been compared with those coming from a special deuterium lamp. It was found that the Hinteregger lamp emitted the highest intensity between 120 and $165 \mathrm{~nm}$, when it was filled with hydrogen of $99.999 \%$ purity flowing slowly through it at a pressure of $800 \mathrm{~Pa}$ $\left(400 \mathrm{~Pa} 1 \mathrm{~s}^{-1}\right.$ ). The discharge produced by a firing voltage of $2100 \mathrm{~V}$ DC runs stably up to a current of $1.0 \mathrm{~A}$. The negative characteristic curve (voltage against current) is shown in figure 2 for the conditions discussed above. The voltage

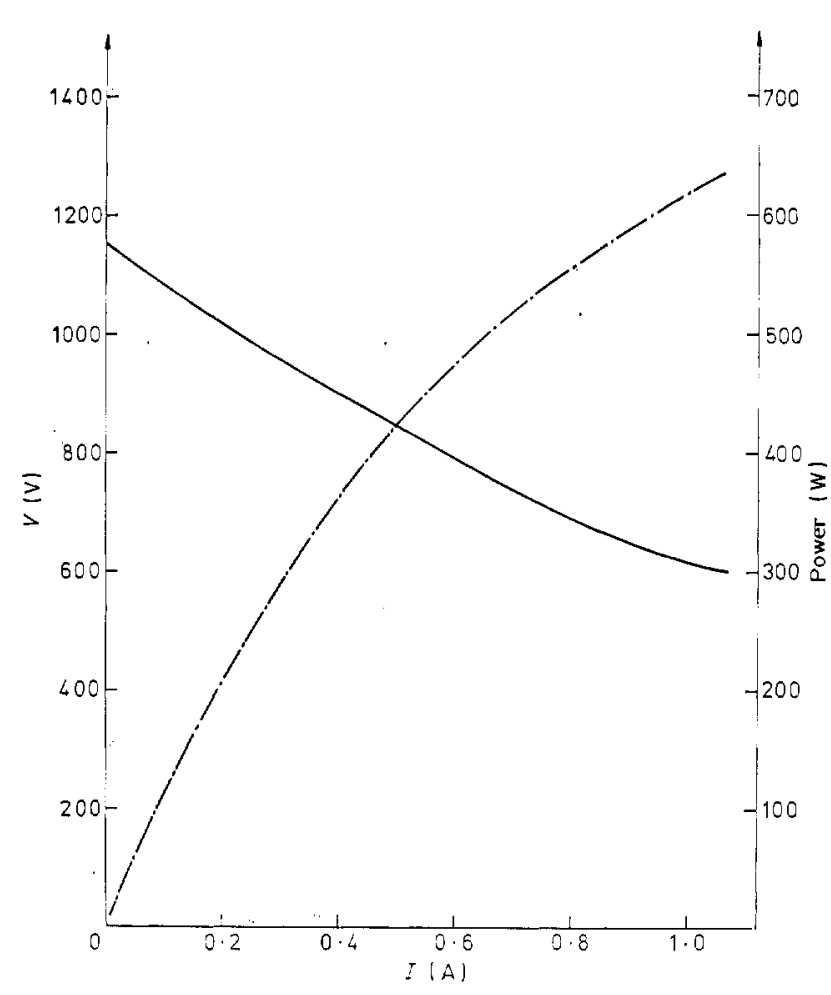

Figure 2 Voltage-current characteristic (full curve) and power (chain curve) of the Hinteregger lamp filled with hydrogen at a pressure of $800 \mathrm{~Pa}$.

decreases from about 1200 to $600 \mathrm{~V}$ with increasing current. In order to obtain stable operation a resistor of $1500 \Omega$ was used in series with the Hinteregger lamp. The electric power of the discharge (up to $600 \mathrm{~W}$ ) is also given in figure 2 . The best conditions for the wavelength range $170-210 \mathrm{~nm}$ are found at a hydrogen pressure of $260 \mathrm{~Pa}$.

It was found experimentally that the Hinteregger lamp, filled with rare gases, yielded an intensity of vuV radiation one to two orders less for all wavelengths between 120 and $210 \mathrm{~nm}$ than under the conditions described above when the lamp was filled with hydrogen. For the condensed discharge in the rare gases, 1000 sparks s ${ }^{-1}$ with a charging voltage of $15 \mathrm{kV}$ at an average current of $0.1 \mathrm{~A}$ and a spark current of $10 \mathrm{~A}$ (RMs) were used. The highest radiation intensity was obtained at a gas pressure of $60 \mathrm{kPa}$ for $\mathrm{Ar}$ and $33 \mathrm{kPa}$ for $\mathrm{Kr}$. It was necessary to purify the rare gases using a cooling trap filled with trichlorethylene and dry ice in the gas circuit and to have the rare gases flowing through the lamp at a high rate $(4 \mathrm{kPa} 1$ $\mathrm{s}^{-1}$ ) in order to achieve the highest intensity. In the wavelength range $180-210 \mathrm{~nm}$, a commercial deuterium lamp with a Suprasil window (Hanau D 102, $50 \mathrm{~W}$ ) yielded an intensity two orders less than the Hinteregger lamp filled with hydrogen.

The quantitative results of the hydrogen spectrum under the conditions discussed above and measured at the output of the exit slit of the monochromator are shown in figure 3. Both slits are $2 \mathrm{~mm}$ wide which means that the radiation has a bandwidth of $6.2 \mathrm{~nm}$; when the two slits are only $1 \mathrm{~mm}$ wide the intensity is reduced to $25 \%$. The absolute scale of figure 3 , which is correct to within a factor of 3 , is calibrated by putting the detector at the output of the exit slit of a second UV monochromator (Bausch and Lomb) combined with either a mercury (HBO 200, Osram) or a xenon (XBO 150, Osram) high-pressure lamp. This calibration was possible because the absolute spectral intensity of both lamps individually and together with the UV monochromator were known from the

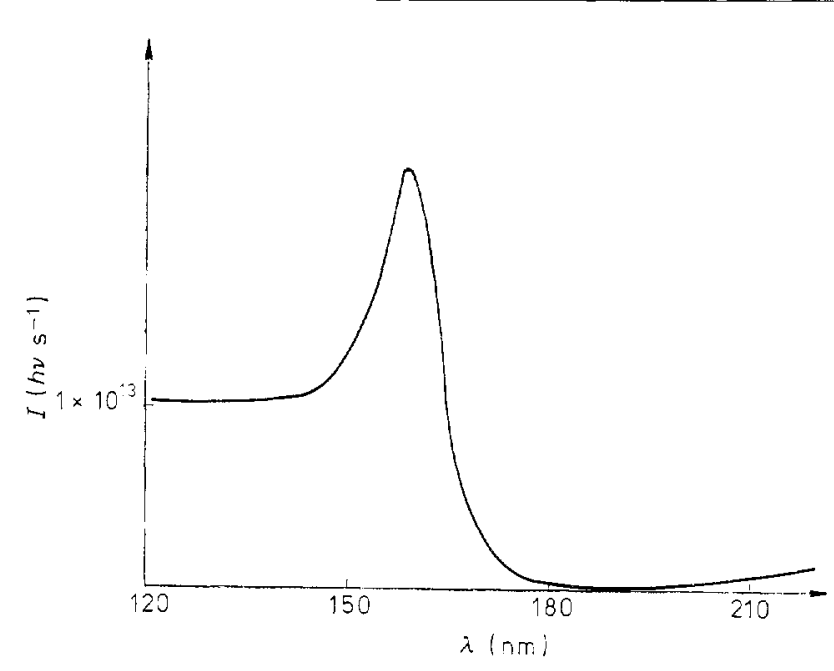

Figure 3 Spectral intensity of radiation behind the $2 \mathrm{~mm}$ wide exit slit of the monochromator.

manufacturers' data. The transmission factor of the Bausch and Lomb monochromator could therefore be calculated, but it has also been experimentally determined as $0.05 \pm 0.03$ at $314 \mathrm{~nm}$ by the use of an interference filter (the different apertures were taken into account).

A comparison of the intensities in different wavelength ranges is possible because the efficiency of the sodium salicylate detector is independent of wavelength (Becker 1963, Samson and Hadded 1974). Additional investigations have been made in order to check this independence: the photoelectron currents in the photoionisation of different atomic vapour beams at different wavelengths have been compared using the well known photoionisation cross sections.

Fine structure cannot be resolved because the radiation with an intensity of the order of $10^{13}$ photons $\mathrm{s}^{-1}$ (figure 3 ) has a bandwidth of $6.2 \mathrm{~nm}$. When the slits of the monochromator are $10 \mu \mathrm{m}$ wide, the intensity distribution shows a true continuum above $165 \mathrm{~nm}$, but below this value the many-line spectrum of molecular hydrogen is seen. With slits of $1 \mathrm{~mm}$ these lines merge into the quasi-continuum shown in figure 3.

\section{Design of the radiation polariser}

There are three different ways of obtaining polarised vur radiation. 
(1) By using synchrotron radiation (Vodar and Romand 1971).

(2) By using prisms, lenses or quarter-wave plates built from material which is doubly refracting (Chandrasekharan and Damany 1971, Johnson 1964, Morris and Abramson 1969, Steinmetz et al 1967).

(3) By reflection or transmission of piles of plates or reflection on metal surfaces at certain angles of incidence (Mcllrath 1968, Matsui and Walker 1970, Rehfeld et al 1973, Robin et al 1966, Walker 1964).

Synchrotron radiation was not available. For the apparatus described here, the second method was selected because of the good polarising power and radiation transmission of doubly refracting optical elements for wavelengths longer than $130 \mathrm{~nm}$. Furthermore, the use of prisms and quarter-wave plates ensures that the vUV radiation beam does not change its direction when it is polarised. In figure 4 the differences in the

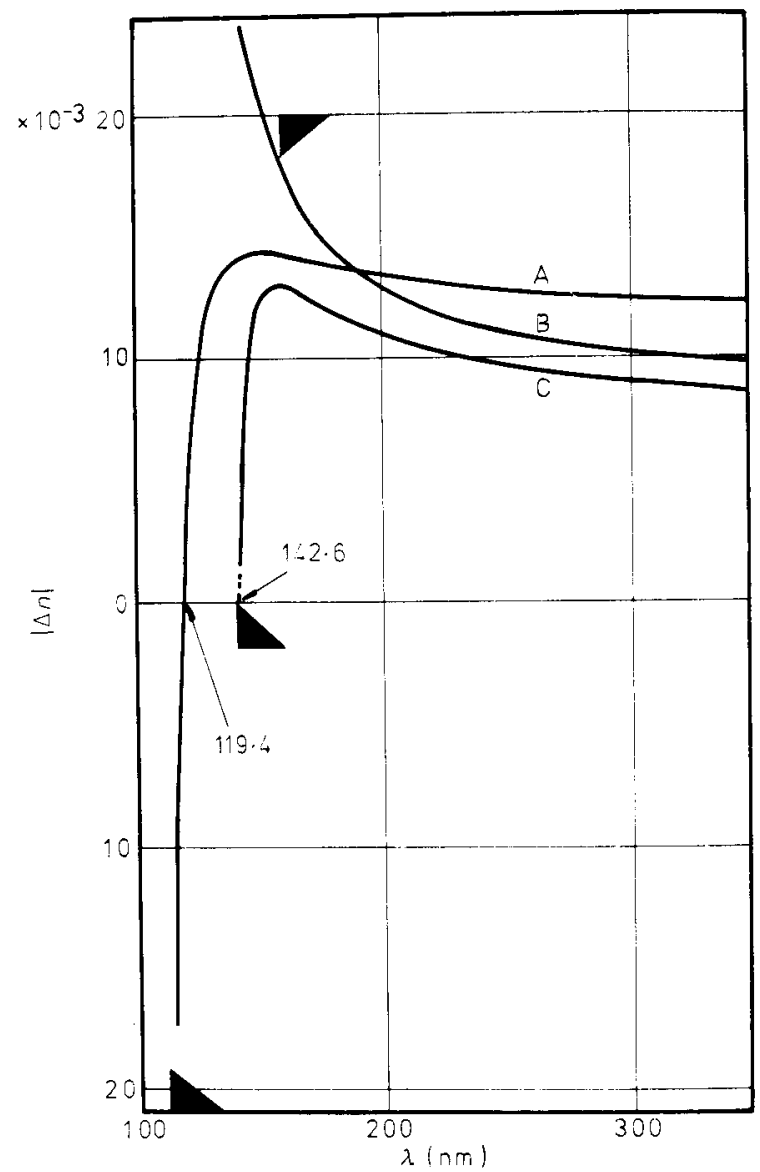

Figure 4 Dispersion of birefringence for $\mathrm{MgF}_{2}$ (curve A), quartz (curve $\mathrm{B}$ ) and sapphire (curve $\mathrm{C}$ ) according to Chandrasekharan and Damany (1969): the difference in the refractive indices. The vertices of the triangles mark the position of the absorption edges (Samson 1967).

refractive indices $n_{\mathrm{e}}$ and $n_{0}$ of quartz, sapphire and $\mathrm{MgF}_{2}$ are given as functions of the wavelength (Chandrasekharan and Damany 1969). This illustrates which materials can be used for building doubly refracting optical elements. $\mathrm{MgF}_{2}$ was chosen because quartz has a high absorption and sapphire loses its birefringence at wavelengths less than $160 \mathrm{~nm}$. As discussed in $\$ 5$, the measurements could only be performed at wavelengths longer than $135 \mathrm{~nm}$. At shorter wavelengths, the transmission of the optical elements was too low.

As shown in figure 1, a linear polariser and analyser of the
Sénarmont double prism type are used $\uparrow$. These double prisms have a cross section of $15 \mathrm{~mm} \times 15 \mathrm{~mm}$ and a length of $30 \mathrm{~mm}$ and have the advantage that a single prism with the same cross section and the same refracting angle of the wedges would be twice as long, resulting in decreased transmission. The shadings in the three parts of the prisms drawn in figure 1 illustrate the positions of the optic axes; in the larger wedges the optic axis lies in the direction of light propagation, in the smaller wedges it is perpendicular to it. Radiation with the electric vector perpendicular to all optic axes, i.e. vertically linear polarised light, passes through the prism unrefracted. Horizontally polarised radiation is refracted away from the optic axis in two beams. However, the angle between the unrefracted and the refracted beams, and this is a disadvantage of all prisms in the VUV region, is very small, between 0 and $3^{\circ}$, depending upon the wavelength (Williams et al 1967). As shown in figure 1 it is therefore necessary to use an LiF lens before the prism in order to ensure that the radiation is parallel when it passes through the prism. The planocylindric lens in front of the prism and the planoconvex lens behind the prism form a rectangular image of the monochromator exit slit at a position where a pinhole separates the radiation beams (which have different kinds of linear polarisation) coming from the prism. The cross sections of all the lenses and the prism are larger than the aperture of the radiation. Because of the high dispersion of LiF in the vuv range (Roessler and Walker 1967) the lenses have to be moved distances of up to $10 \mathrm{~cm}$, so that the exit slit and the pinhole are always at the focus of the lenses.

The linearly polarised radiation emerging from the pinhole is circularly polarised by an $\mathrm{MgF}_{2}$ quarter-wave double plate; two plates with diameters of $30 \mathrm{~mm}$ have an angular difference of $90^{\circ}$ between their optic axes and a thickness difference such that the optical path difference for the two linear polarisation states is $\lambda / 4$ at $\lambda=150 \mathrm{~nm}$ (for details see $\$ 5$ ). In front of and behind the quarter-wave double plate are two LiF lenses. The first produces parallel radiation and the second focuses the light on to the atomic beam where the photoelectrons are produced. There the radiation beam has a diameter of $5 \mathrm{~mm}$ and an angular divergence of $\pm 2.5^{\circ}$. The radiation analyser has the same construction as the polariser, except that there is no quarter-wave plate in the radiation beam.

It is known (Taylor et al 1965) that contamination by water or organic oils can reduce the transmission of $\mathrm{LiF}$ and $\mathrm{MgF}_{2}$ optical elements in the VUV range; it is thus necessary to have clean vacuum conditions. All the polarising optics described above are therefore separated from the atomic vapour beam by an $\mathrm{LiF}$ window: a turbomolecular pump evacuates the vacuum chamber to a pressure of better than $4 \times 10^{-4} \mathrm{~Pa}$. Only ungreased Viton $O$-rings are used, and bellows seals are used in the manipulators for moving the lenses, prisms and the quarter-wave plate. The linear and circular polarisers, together with their lenses, are mounted on one optical bench about $1 \mathrm{~m}$ long. Glass filters can be moved into the radiation beam at different places in order to stop the UV and VUV, so that the component of visible background radiation can be measured. The positions of all lenses and the total optical path, of length $3.5 \mathrm{~m}$, are adjusted with a $\mathrm{He}-\mathrm{Ne}$ laser or a halogen lamp.

5 Results of polarisation and transmission of the polariser Partially polarised radiation can be considered to be a superposition of unpolarised and totally polarised components. The

$\dagger$ All optical elements were built by Fa. Halle, Berlin, West Germany. 
total polarisation (commonly called degree of polarisation) is defined as

$$
P_{\text {tot }}=I_{\text {pol }} / I_{\text {tot }}
$$

where $I_{\mathrm{poI}}$ is the intensity of the totally polarised component, and $I_{\text {tot }}$ is the total intensity. In our case, $P_{\text {tot }}$ is the linear polarisation of the light between the linear polariser and the quarter-wave plate and is experimentally determined by use of a second polariser as an analyser. Then one obtains (Heinzmann 1970)

$$
P_{\text {tot }}=\left[\left(I_{\mid i}-I_{\perp}\right) /\left(I_{||}+I_{\perp}\right)\right]^{1 / 2} .
$$

$I_{\mid}$and $I_{\perp}$ are the intensities of radiation measured behind the analyser when the axes of the analyser and the polariser are respectively parallel and perpendicular and when the axis of the quarter-wave plate is parallel to the axis of the linear polariser. The square root in equation (2) arises from the fact that the analyser is also not ideal. Equation (2) is only correct if the polariser and the analyser have the same construction and properties and if the polarisation of the radiation coming from the monochromator is low compared with that behind the prism. A test measurement yielded a polarisation of lower than $4 \%$ for the radiation coming from the exit slit at $158 \mathrm{~nm}$.

In order to be sure that the linear polariser and the analyser are working correctly, the intensity of the radiation transmitted through both has to be measured as a function of the angle $\phi$ between their axes. It is necessary for this intensity to have the form $I=I_{\perp}+\left(I_{\|}-I_{\perp}\right) \cos ^{2} \phi$, which means that the measured intensity $I$ is not influenced by a shift of the radiation beam over the pinhole (see figure 1) when either the polariser or the analyser is rotated. The results are given in figure 5 for a

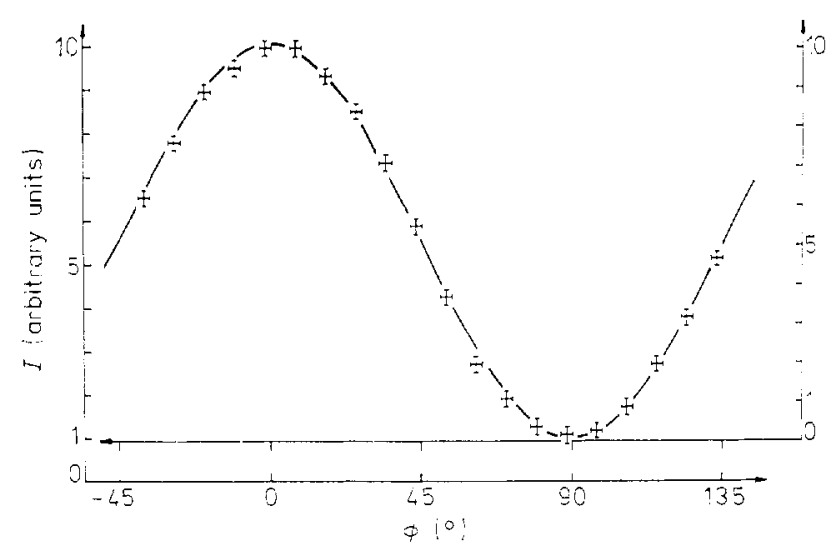

Figure 5 Intensity of radiation $I$ passing through the polariser and analyser as a function of the angle $\phi$ between their axes: experimental values (crosses) and fit according to $I=I_{\perp}+\left(I_{\|}-I_{\perp}\right) \cos ^{2} \phi$ for $140 \mathrm{~nm}$ (left-hand scale) and all wavelengths longer than $150 \mathrm{~nm}$ (right-hand scale).

wavelength of $140 \mathrm{~nm}$ where $P_{\text {tot }}=0.90$ and for all wavelengths longer than $150 \mathrm{~nm}$ where $P_{\text {tot }}=0.99$. The clearly evident $\cos ^{2}$ function also verifies that the efficiency of the sodium salicylate detector is independent of the intensity of the incoming VUV radiation.

Behind the quarter-wave plate, $I_{\mathrm{por}}$ is the intensity of the elliptically polarised radiation. If the Stokes parameter formalism is used (Born and Wolf 1959), the circular polarisation of the radiation in the position of the atomic vapour beam is given by

$$
P=I_{3} / I_{\text {tot }}=P_{\text {tot }}\left(I_{3} / I_{\mathrm{pol}}\right) .
$$

$I_{3}$, the third Stokes parameter, is the difference between the intensities of right-handed and left-handed circularly polarised light, if this means of superposition is selected. With two orthogonal linear oscillations one obtains

$$
I_{3}=2 a b \sin \delta
$$

where $a$ and $b$ are the amplitudes and $\delta$ is the phase difference. In the rotated coordinate system of the principal axes of the ellipse, $I_{3}$ and $I_{\text {pol }}$ are given by

$$
I_{3}=2 A B \quad I_{\mathrm{pol}}=A^{2}+B^{2}
$$

where $A$ and $B$ are the semi-axes of the ellipse.

Then according to equations (3), (5) and (2), the circular polarisation is

$$
\begin{aligned}
P & =P_{\text {tot }}\left[2 A B_{i}\left(A^{2}+B^{2}\right)\right] \\
& =\left(\frac{I_{\|}-I_{\perp}}{I_{\|}+I_{\perp}}\right)^{1 / 2} \frac{2\left(I_{\max }-I_{\perp}\right)^{1 / 2}\left(I_{\min }-I_{\perp}\right)^{1 / 2}}{I_{\max }-I_{\perp}+I_{\min }-I_{\perp}} .
\end{aligned}
$$

$I_{\max }$ and $I_{\min }$ are the highest and lowest intensities obtained when the analyser prism is rotated around the direction of propagation of light when the linear polariser and the quarterwave plate have an angular difference of $45^{\circ}$ between their axes. An additional test was to show that $I_{\max }+I_{\min }=I_{\mid}+I_{-}$, which means that the transmission of the quarter-wave plate is independent of the position of its axis.

Figure 6 shows the results of measuring the circular polarisation with radiation in the wavelength range $140-300 \mathrm{~nm}$. The decline of $P$ to shorter wavelengths at $140 \mathrm{~nm}$ is due to the decrease in the birefringence of $\mathrm{MgF}_{2}$ (see figure 4 ); it is then

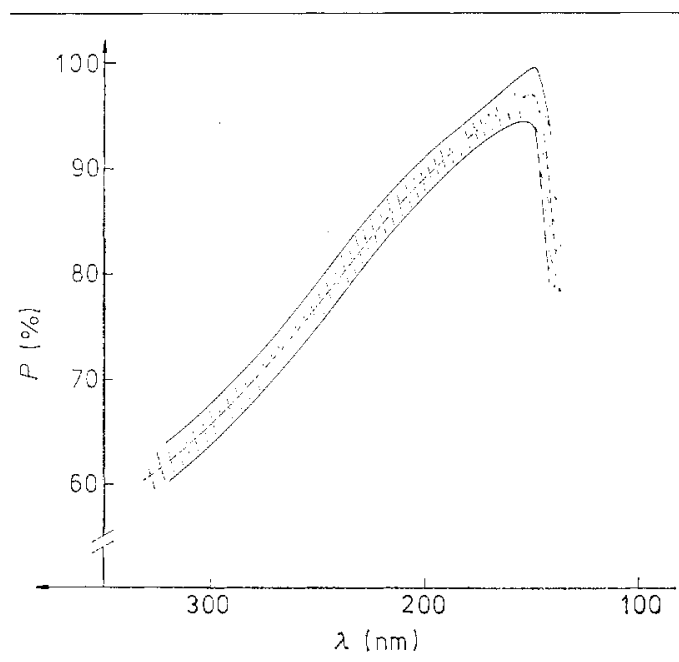

Figure 6 Circular polarisation of radiation with error limits (hatched area).

no longer possible to separate completely the two differently linearly polarised radiation beams by the pinholes (see figure 1) unless the pinholes were to be partially closed, causing a sharp reduction in the intensity of the transmitted radiation.

For wavelengths longer than $150 \mathrm{~nm}$, the polarisation efficiency of the linear polariser is better than $99 \%$. The decline of $P$ at longer wavelengths is caused only by the properties of the quarter-wave plate. This wavelength dependence is described by equation (4) with $\delta=2 \pi \Delta / \lambda$ where $\Delta$ is the optical path difference for the two linear polarisation states and is $\lambda / 4$ at $\lambda=150 \mathrm{~nm}$, as pointed out in $\S 4$. $\Delta$ is proportional to the difference between the refractive indices $\Delta n$ shown in figure 4 . The polarisation results of figure 6 can be used to calculate the path difference $\Delta$. It is tabulated together with $\Delta n$ in table 1 . The wavelength dependence of $\Delta n$ is in good agreement with the results of Chandrasekharan and Damany (1969) shown in figure 4 . 


\section{Table 1}

\begin{tabular}{lll}
\hline $\begin{array}{l}\text { Wavelength } \\
(\mathrm{nm})\end{array}$ & $\begin{array}{l}\text { Path difference } \\
(\mathrm{nm})\end{array}$ & $\begin{array}{l}\Delta n \\
\text { (arbitrary units) }\end{array}$ \\
\hline 150 & $75 \pm 2 \cdot 5$ & $1.00 \pm 0.03$ \\
175 & $71 \pm 3 \cdot 0$ & $0.95 \pm 0.03$ \\
200 & $71.9 \pm 2 \cdot 5$ & $0.96 \pm 0.03$ \\
225 & $72 \cdot 6 \pm 2 \cdot 6$ & $0.97 \pm 0.03$ \\
250 & $71 \cdot 2 \pm 2 \cdot 6$ & $0.95 \pm 0.03$ \\
275 & $70 \pm 2 \cdot 5$ & $0.93 \pm 0.03$ \\
300 & $69 \cdot 2 \pm 2 \cdot 6$ & $0.92 \pm 0.03$ \\
325 & $68.9 \pm 2.9$ & $0.92 \pm 0.04$ \\
\hline
\end{tabular}

In order to determine the transmission of the optical elements, the radiation intensities behind these have been measured and compared with the intensities measured directly at the exit slit of the monochromator (given in figure 3). It was found that the double prism has a transmission of $50 \%$ at $158 \mathrm{~nm}$ and $38 \%$ at $142 \mathrm{~nm}$ for linearly polarised incident light. Each lens and the quarter-wave double plate possess a transmission of $65 \%$ at $158 \mathrm{~nm}$ and $56 \%$ at $142 \mathrm{~nm}$. The linear polariser, the combination of the prism with two lenses, thus has a transmission of $21 \%$ at $158 \mathrm{~nm}$ and $12 \%$ at $142 \mathrm{~nm}$ and the circular polariser (quarter-wave plate with two lenses) has a transmission of $27 \%$ at $158 \mathrm{~nm}$ and $18 \%$ at $142 \mathrm{~nm}$. Thus in the atomic beam region, the maximum numbers of photons $\mathrm{s}^{-1}$ are about $4 \times 10^{11}$ at $158 \mathrm{~nm}$ and $6 \times 10^{10}$ at $142 \mathrm{~nm}$, and at the detector the numbers are $2 \times 10^{10}$ at $158 \mathrm{~nm}$ and $10^{\circ}$ at $142 \mathrm{~nm}$.

\section{Conclusions}

The apparatus described above produces a circularly polarised vUV radiation beam of high intensity. At the atomic vapour beam, where the photoelectrons are produced, this intensity amounts to about $4 \times 10^{11}$ photons $\mathrm{s}^{-1}$ at $158 \mathrm{~nm}$, and about $6 \times 10^{10}$ at $142 \mathrm{~nm}$ with a bandwidth of $6.2 \mathrm{~nm}$. Radiation with half the bandwidth has a quarter of this intensity. The measurements of the spin polarisation of photoelectrons emitted from lead and thallium atoms (Heinzmann 1977, Heinzmann et al $1975,1976)$ have shown that the lowest intensity of radiation which may be used to measure the spin polarisation of photoelectrons successfully is at least $10^{10}$ photons $\mathrm{s}^{-1}$ for a photoionisation cross section of not lower than $10^{-22} \mathrm{~m}^{2}$. The circular polarisation should not be less than about $80 \%$. These numbers should be a guide for all experiments including those using synchrotron radiation, where the spin polarisation of photoelectrons emitted from atoms or molecules is to be measured.

\section{Acknowledgments}

The author wishes to express his gratitude to Professor $\mathbf{J}$ Kessler for stimulating discussions, and for the financial support for the experiment described in the paper. He is grateful to Dr C B Lucas for reading the manuscript and making valuable suggestions. He gratefully acknowledges the technical assistance of $\mathbf{H}$ Gerberon in constructing the apparatus and of $\mathrm{H}$ Heuer and $\mathrm{E}$ Kuhlmann in evaluating the measurements.

\section{References}

Adriaens M R and Feuerbacher B 1971 Appl. Opt. 10 958-9

Becker A 1963 Max Planck Institut für Physik und Astrophysik, München, Report No. MPI-PA-18/63
Born M and Wolf E 1959 Principles of Optics (London: Pergamon) pp 542-51

Branch J D, Heddle D W O and Mogridge M J H 1971 J. Phys. E: Sci. Instrum. 4 9-12

Chandrasekharan V and Damany H 1969 Appl. Opt. 8 671-5 Chandrasekharan V and Damany H 1971 Appl. Opt. 10 681-2

Christensen R L and Potter R J 1963 Appl. Opt. 2 1049-54

Comes F J 1960 Z. Instrumentenkunde 68 69-76

Davis D and Braun W 1968 Appl. Opt. 7 2071-4

Fano U 1969 Phys. Rev. 178 131-6

Heinzmann U 1970 Diplomarbeit Universität Karlsruhe (W Germany) 13-18

Heinzmann U 1977 submitted to J. Phys. B: Atom. Molec. Phys.

Heinzmann U, Heuer H and Kessler J 1975 Phys. Rev. Lett. 34 441-4, 710

Heinzmann U, Heuer H and Kessler J 1976 Phys. Rev. Lett. 36 1444-7

Heinzmann U, Kessler J and Lorenz J 1970 Z. Phys. 240 42-61

Huffman R E, Larrabee J C and Tanaka Y 1965 Appl. Opt. 4 1581-8

Huffman R E, Tanaka Y and Larrabee J C 1961 Proc. 5th Int. Conf. on Phenomena in Ionized Gases, München

(Amsterdam: North-Holland) pp 1938-44

Johnson M C 1969 Rev. Sci. Instrum. 40 311-5

Johnson W C 1964 Rev. Sci. Instrum. 35 1375-6

Johnston R G and Madden R P 1965 Appl. Opt. 4 1574-80

Kessler J 1976 Polarized Electrons (Berlin: Springer-Verlag) pp 125-39

Matsui A and Walker W C 1970 J. Opt. Soc. Am. 60 64-5

McIlrath T J 1968 J. Opt. Soc. Am. 58 506-10

Morris G C and Abramson A S 1969 Appl. Opt. 8 1249-50

Paresce F 1975 Appl. Opt. $142823-4$

Rehfeld N, Gerhardt U and Dietz E 1973 Appl. Phys. 1

229-32

Robin M B, Kuebler N A and Yoh-Han Pao 1966 Rev. Sci. Instrum. 37 922-4

Roessler D M and Walker W C 1967 J. Opt. Soc. Am. 57 $835-6$

Samson J A R 1967 Techniques of VUV-Spectroscopy (New York: Wiley) pp 180-4

Samson J A R and Haddad G N 1974 J. Opt. Soc. Am. 64 1346-7

Steinmetz D L, Phillips W G, Wirick M and Forbes F F 1967 Appl. Opt. 6 1001-4

Taylor R G, Chubb T A and Kreplin R W 1965 J. Opt. Soc. Am. 55 1078-81

Vodar B and Romand J 1971 Proc. 3rd Int. Conf. on VUV Radiation Physics, Tokyo (Tokyo: Physical Society of Japan) 31aA1-1, pp 1-7

Walker W C 1964 Appl. Opt. 3 1457-9

Williams M W, MacRae R A and Arakawa E T 1967

J. Appl. Phys, 38 1701-5

Journal of Physics E: Scientific Instruments 1977 Volume 10 Printed in Great Britain (C) 1977 